\title{
Soft Inverse Semigroup
}

\author{
Bingshen $\operatorname{Lan}^{1, a}$ \\ ${ }^{1}$ Engineering and Technical College of Chengdu University of Technology, China \\ a17935389@qq.com
}

Keywords: Soft inverse semigroup; Soft morphism; Soft group congruence.

\begin{abstract}
In this paper, the concepts of soft inverse semigroup, soft normal semigroup on inverse semigroup, and soft morphism are given. Some characterizations and results are obtained. Next, the definitions of soft group congruence on inverse semigroup is introduced, the relationship between soft group congruence and soft normal semigroup on inverse semigroup are studied more.
\end{abstract}

\section{Introduction}

Soft set theory which deals with uncertainty problem in many fields is given by Molodstov in 1999[1]. Aktas H, et al give the concept soft group.[2], Ali discusses soft binary relationon and soft equivalence on set[3], Sezgin A, Atagün A O gives the concept normalistic soft group[4] . In this paper, based on soft sets, soft inverse semigroup and soft congruence on inverse semigroup are syudied.

$(F, A)$ is called soft set on $U$, such that $F: A \rightarrow P(U), P(U)$ is the power set of $U$. [1]

$(\rho, A)$ is called soft binary relation on $U \times U$, such that $(\rho, A)$ is soft set on $U \times U .[3]$

If $x$ is an element of a semigroup $S, x^{-1}$ is called an inverse of $x$, such that $x x^{-1} x=x, x^{-1} x x^{-1}=x^{-1}$.

A semigroup $S$ with set $E(S)$ of idempotents, if for any $x, y \in S$ suah that $x x^{-1}=x^{-1} x, x x^{-1} y y^{-1}=y y^{-1} x x^{-1}$, then $S$ is called an inverse semigroup [5].

A subsemigroup $T$ of an inverse semigroup $S$ is said to be self-conjugate if for any $x \in S$, such that

$t \in T \Rightarrow \forall x \in S, \quad x^{-1} t x \in T .[5]$

A subsemigroup $T$ of inverse semigroup $S$ is said to be normal if $T$ such that the following three conditions.

(1) $T$ contains all the idempotents of $S, E(S) \subseteq T$;

(2) $T$ is an inverse subsemigroup os $S$;

(3) $T$ is self-conjugate. [5]

A left (right) compatible equivalence is called a left (right) congruence. A compatible equivalence is called congruence. [5]

If $\rho$ is a congruence on semigroup $\mathrm{S}$, then we can define a binary operation on the quotient set $S / \rho$ as follows:

$(a \rho)(b \rho)=(a b) \rho$.

It is easily to prove that $S / \rho$ is a semigroup. [5]

If $\rho$ is a congruence on inverse semigroup $S, S / \rho$ is a group, then $\rho$ is saided a group congruence on inverse semigroup $S$.

Proposition1.1 ${ }^{[5]} T$ is a normal subsemigroup of inverse semigroup $S$, then

$$
\rho_{\mathrm{T}}=\left\{(a, b) \in S \times S \mid a b^{-1} \in T\right\}
$$


is a group congruence on $\mathrm{S}$. Conversely, let $\rho$ be a group congruence on inverse semigroup $\mathrm{S}$, then

$$
T_{\rho}=\operatorname{ker} \rho=\bigcup_{e \in E} e \rho=\left\{s \in S \mid s \rho=1_{S / \rho}\right\}
$$

is a normal subsemigroup of inverse semigroup $\mathrm{S}$.

\section{Soft Inverse Semigroup}

Definition 2.1 Let $(F, A)$ be soft set on an inverse semigroup $S$, for each a of $A, F(a)$ is an inverse subsemigroup of $S$, then $(F, A)$ is called soft inverse semigroup on $S$.

If $F(a)$ is a normal subsemigroup of $S$, then $(F, A)$ is called soft normal semigroup on $S$.

Example2.1 Let $S=\{o, a, b, c, d, e, f\}$ with multiplication table

\begin{tabular}{c|ccccccc} 
& $\mathrm{o}$ & $\mathrm{a}$ & $\mathrm{b}$ & $\mathrm{c}$ & $\mathrm{d}$ & $\mathrm{e}$ & $\mathrm{f}$ \\
\hline $\mathrm{o}$ & $\mathrm{o}$ & $\mathrm{o}$ & $\mathrm{o}$ & $\mathrm{o}$ & $\mathrm{o}$ & $\mathrm{o}$ & $\mathrm{o}$ \\
$\mathrm{a}$ & $\mathrm{o}$ & $\mathrm{a}$ & $\mathrm{o}$ & $\mathrm{c}$ & $\mathrm{o}$ & $\mathrm{a}$ & $\mathrm{c}$ \\
$\mathrm{b}$ & $\mathrm{o}$ & $\mathrm{o}$ & $\mathrm{b}$ & $\mathrm{o}$ & $\mathrm{d}$ & $\mathrm{b}$ & $\mathrm{d}$ \\
$\mathrm{c}$ & $\mathrm{o}$ & $\mathrm{o}$ & $\mathrm{c}$ & $\mathrm{o}$ & $\mathrm{a}$ & $\mathrm{c}$ & $\mathrm{a}$ \\
$\mathrm{d}$ & $\mathrm{o}$ & $\mathrm{d}$ & $\mathrm{o}$ & $\mathrm{b}$ & $\mathrm{o}$ & $\mathrm{d}$ & $\mathrm{b}$ \\
$\mathrm{e}$ & $\mathrm{o}$ & $\mathrm{a}$ & $\mathrm{b}$ & $\mathrm{c}$ & $\mathrm{d}$ & $\mathrm{e}$ & $\mathrm{f}$ \\
$\mathrm{f}$ & $\mathrm{o}$ & $\mathrm{d}$ & $\mathrm{c}$ & $\mathrm{b}$ & $\mathrm{a}$ & $\mathrm{f}$ & $\mathrm{e}$
\end{tabular}

It is easy to show that $S$ is an inverse semigroup.

$A=\{1,2,3,4,5,6,7\}$, let

$$
\begin{gathered}
F(1)=\{a\}, F(2)=\{e, f\}, \mathrm{F}(3)=\{o, e, f\}, F(4)=\{o, a, b, e\}, \\
F(5)=\{o, a, b, c, d\}, F(6)=\{o, a, b, c, d, e\}, F(7)=\{o, a, b, c, d, e, f\} .
\end{gathered}
$$

And $F(1), F(2), \mathrm{F}(3), F(4), F(5), F(6), F(7)$ are inverse subsemigroups of $S$. So, $(F, A)$ is soft inverse semigroup on $S$.

Example2.2 let $S$ as same as Example2.1, $B=\{\mathbf{4 , 6 , 7}\}$

Let $F(4)=\{o, a, b, e\}, F(6)=\{o, a, b, c, d, e\}, F(7)=\{o, a, b, c, d, e, f\}$.

It is easy to see that

$$
F(4)=\{o, a, b, e\}, F(6)=\{o, a, b, c, d, e\}, F(7)=\{o, a, b, c, d, e, f\}
$$

are normal subsemigroup of $S$, So, $(F, A)$ is soft normal semigroup on $S$. It is clear that if $(F, B)$ is soft normal semigroup on inverse semigroup $S$, then $(F, B)$ is Soft inverse semigroup on $S$.

Definition 2.3 Let $(F, A),(H, B)$ be soft inverse semigroup on an inverse semigroup $S$, if

$A \subset B$;

$\forall x \in \mathrm{A}, F(X)$ is subsemigroup of $H(x)$

Then, $(F, A)$ is called sofe subsemigroup of $(H, B)$.

It is clear that $(F, B)$ (Example2.2) is soft subsemigroup of $(F, A)$ ( Example2.1).

Proposition2.1 If $(F, A),(G, A)$ are soft inverse semigroup on inverse semigroup $S$, then $(F, A) \bigcap(G, A)$ is soft inverse semigroup on $S$.

Let $(U, A)=(F, A) \cap(G, A)$, for each $a$ of $A, U(a)=F(x)$, or $G(x)$, it is clear that $(F, A) \cap(G, A)$ is soft inverse semigroup. And $(F, A) \cap(G, A)$ is soft $\operatorname{subsemigroup~of~}(F, A)$ or $(G, A)$.

Proposition 2.2 If $(F, A),(G, B)$ are soft inverse semigroup on inverse semigroup $S, A \cap B=\Phi$, 
then $(F, A) \bigcup(G, B)$ is soft inverse semigroup on $S$.

Let $(U, C)=(F, A) \bigcup(\mathrm{G}, B), C=A \cup B$, for each $a \in C$,

$U(a)=\left\{\begin{array}{l}F(a), a \in A-B \\ G(a), a \in B-A\end{array}\right.$,

it is clear that $(F, A) \bigcup(G, B)$ is soft inverse semigroup. And $(F, A),(G, B)$ are soft subsemigroup of $(F, A) \bigcup(G, B)$.

Definition $2.4^{[6]}$ Let $(F, A)$ be soft semigroup on semigroup $\mathrm{S},(G, B)$ be soft semigroup on semigroup $\mathrm{T}, \quad f: S \rightarrow T, g: A \rightarrow B$ if

$\mathrm{f}$ is morphism;

$\mathrm{g}$ is map;

$\forall x \in A, f(F(x))=G(g(x))$.

Then, $(f, g)$ is called soft morphism from $(F, A)$ to $(G, B)$. It denoted by

$(f, g):(F, A) \rightarrow(G, B)$

If, $\mathrm{f}$ is isomorphic morphism and $\mathrm{g}$ is bijection, $(f, g)$ is called soft isomorphism from $(F, A)$ to $(G, B)$.

Example2.3

$I_{x}=\left\{A=\left(\begin{array}{l}1 \\ 1\end{array}\right), B=\left(\begin{array}{l}2 \\ 2\end{array}\right), \quad C=\left(\begin{array}{l}1 \\ 2\end{array}\right), D=\left(\begin{array}{l}2 \\ 1\end{array}\right), \quad E=\left(\begin{array}{ll}1 & 2 \\ 1 & 2\end{array}\right), F=\left(\begin{array}{ll}1 & 2 \\ 2 & 1\end{array}\right), \quad 0\right\}$

consist of all partial one-one maps of $\mathrm{X}, \mathrm{O}$ are denoted by empty map. The operation of $\mathrm{I}_{\mathrm{X}}$ :

$\forall H, G \in I_{X},(x, y) \in H G \Leftrightarrow \exists z \in X,(x, z) \in H,(z, y) \in G$.

Let, $N=\{1,2,3,4,5,6,7\}$,

$G(1)=\{A\}, G(2)=\{E, F\}, \mathrm{G}(3)=\{O, E, F\}, G(4)=\{O, A, B, E\}$,

$G(5)=\{O, A, B, C, D\}, G(6)=\{O, A, B, C, D, E\}, G(7)=\{O, A, B, C, D, E, F\}$

Clearly, $(G, N)$ is soft inverse semigroup on inverse semigroup $\mathrm{I}_{\mathrm{x}}$. Soft inverse semigroup $(F, A)$ and soft inverse semigroup $(G, N)$ are soft isomorphism.

\section{Soft Group Congruence on Inverse Semigroup}

Definition 3.1 $S$ is an inverse semigroup, let $(\rho, A)$ be soft binary relation on $S \times S$, for any $\alpha \in A, \rho(\alpha)$ is a group congruence on an inverse semigroup $S$, then $(\rho, A)$ is called soft group congruence on an inverse semigroup $S$.

Proposition3.1 let ( $F$, A ) is soft normal semigroup of inverse semigroup $S$, for any $a \in A$, let $\rho(a)=\rho_{F(a)}=\left\{(a, b) \in S \times S \mid a b^{-1} \in \mathrm{F}(\mathrm{a})\right\}$

Then $(\rho, \mathrm{A})$ is soft group congruence on E-inversive semigroup $\mathrm{S}$.

Conversely, let $(\rho, \mathrm{B})$ is soft group congruence on E-inversive semigroup $\mathrm{S}$, then, for any $b \in B$, let

$$
F(b)=\mathrm{T}_{\rho(b)}=\operatorname{ker} \rho(b)=\bigcup_{e \in E} e \rho(b)=\left\{s \in S \mid \mathrm{s} \rho=1_{S / \rho(b)}\right\}
$$

Then $(F, B)$ is soft normal semigroup on inverse semigroup $S$.

We can easily show Proposition3.1 from Proposition1.1.

Proposition3.2 ${ }^{[6]}$ Let $S, T$ are semigroups, $f: S \rightarrow T$ is semigroup morphism, if $\rho$ is 
congruence on $\mathrm{S}$, then

$$
f(\rho)=\{(\mathrm{f}(\mathrm{a}), \mathrm{f}(\mathrm{b})) \in \mathrm{T} \times \mathrm{T} \mid a \rho b\}
$$

is congruence on $T$.

Proposition3.3 ${ }^{6]}$ Let $S, T$ are semigroups, $f: S \rightarrow T$ is semigroup morphism, if $(\rho$, A $)$ is soft congruence on $\mathrm{S}$, then

$(f(\rho), \mathrm{A}): f(\rho)(\mathrm{a})=\mathrm{f}(\rho(\mathrm{a})), \forall \mathrm{a} \in \mathrm{A}$

is soft congruence on $\mathrm{T}$.

Proposition3.4 Let $S$ is an inverse semigroup, $G$ is a group, $f: S \rightarrow G$ is semigroup morphism,and $G$ is the maximum group morphic image of $\mathrm{S}$, if $(\rho, \mathrm{A})$ is soft group congruence on $\mathrm{S}$, then $(f(\rho), \mathrm{A})$ ) is soft group congruence on G. Soft quotient soft $G /(f(\rho), \mathrm{A}), S /(\rho, \mathrm{A})$ are isomorphism.

proof From Proposition3.5, $(f(\rho), \mathrm{A}))$ is soft group congruence on $G$. Let

$$
\begin{aligned}
& \forall a \in A, g: G / f(\rho(a)) \rightarrow S / \rho(a), \\
& \forall a \in A, s \in S, g:(f(s) \rho(a)) \rightarrow s \rho(a),
\end{aligned}
$$

Then $\mathrm{g}$ is one-one, since

$$
\begin{aligned}
& \forall s_{1}, s_{2} \in S, \quad g:\left(f\left(s_{1}\right) \rho(a)\right) \rightarrow s_{1} \rho(a), \quad g:\left(f\left(s_{2}\right) \rho(a)\right) \rightarrow s_{2} \rho(a) \\
& \text { if } s_{1} \rho(a)=s_{2} \rho(a) \Rightarrow\left(s_{1}, s_{2}\right) \in \rho(a), \text { from } \\
& \left(f\left(s_{1}\right), f\left(s_{2}\right)\right) \in f(\rho(a)) \Rightarrow f\left(s_{1}\right) f\left(\rho(a)=f\left(s_{2}\right) f(\rho(a),\right.
\end{aligned}
$$

$\mathrm{g}$ is monomorphism, clearly $\mathrm{g}$ is epimorphism, So $\mathrm{g}$ is isomorphism.

Soft quotient soft $G /(f(\rho)$, A $), S /(\rho$, A $)$ is isomorphism.

\section{References}

[1] Molodtsov D. Soft set theory-first results, Comput Math Appl. 1999, 37(4):19-31.

[2] Aktas H, et al. Soft sets and soft groups, Inform Sci. 2007,177 (13):2726-2735.

[3] Ali M I. A note on soft sets, rougf sets and fuzzy soft sets, Applied Soft Computing. 2011, 11(4):3329-3332.

[4] Sezgin A, Atagün A O. Soft groups and normalistic soft groups, Comput Math Appl. 2011, 62(2):685-698.

[5] Howie J. M, Fundamentals of Semigroup Theory, Clarendon Press, Oxford, 1995.

[6] Li Wenting et al, Soft congruence relations on groups, Fuzzy Systems and Mathematics. 2014, 28(2):76-81. 